\title{
Interactive comment on "Ceilometer evaluation of the East Mediterranean summer boundary layer height - first study of two Israeli sites" by L. Uzan et al.
}

\author{
L. Uzan et al. \\ Leenesu@gmail.com \\ Received and published: 20 June 2016
}

Thank you very much for the constructive comments. Apart of the suggestions to improve the manuscript structure and the technical comments, we are examining the option to produce the quantitative results requested under the time limit of the AMT publication. For now, our overall response is given in the table below:

1.Comment: The paper must be steamlined and better structured-combine section 2 , section 3 and first part of section 4. Response:Comment accepted.

2.Comment: The WCT can be considered as well established. Response: We are considering the option of removing the WCT description. 
3.Comment: Second part of section 4 should be discussed as results. Response: Comment accepted.

4. Comment: Language should be improved by a native speaker . Response: Comment accepted.

5. Comment: The ceilometers should validate the model and not the other way around.

Interactive Response: Comment accepted.

comment

6.Comment: Give references to all models. Response: Comment accepted.

7.Comment: Extend the discussion to have sufficient material for a separate section. Response: Comment accepted.

8.Comment: Dedicate a section of PM10 and enlarge the investigated range bin, explain calibration process and the quantity of "backscatter intensity". If it is too early for ultimate results they should be labeled "preliminary" and further steps should be outlined. Response: Comment accepted.

9. Comment: Extend the results with a more detailed discussion and highlight the value of the results. Response: Comment accepted.

10. Comment: The result should include an outlook and identification of open questions and the benefit of more data. Response: Comment accepted.

10. Technical comments: A list of 30 technical comments and question was given. Response: Thank you for the "easy to follow and find" arrangement of the comments. The manuscript will be corrected accordingly.

With most respect,

Leenes Uzan on behalf of the co-authors

Interactive comment on Atmos. Meas. Tech. Discuss., doi:10.5194/amt-2016-85, 2016. 